\title{
Experimental and Numerical Research on 3-D Crack Growth in Rock-like Material Subjected to Uniaxial Tension
}

\author{
Lei Yang ${ }^{1}$, Yujing Jiang ${ }^{2}$, Shucai $\mathrm{Li}^{3}$ and $\mathrm{Bo} \mathrm{Li}^{4}$
}

\begin{abstract}
Uniaxial tension experiments on pre-cracked rock-like samples and the related numerical simulations using FRANC3D were performed to investigate the growth mechanism of 3-D cracks in rocks under tension as well as the strength and failure behavior of samples. Experimental results indicate that the geometrical characteristics of pre-existing cracks, including crack dip angle, crack spacing and crack intensity, have significant effects on the strength and failure modes of the samples. Failure of samples resulted from the propagation of a single crack and the coalescence of multiple cracks if the spacing between adjacent cracks is less than the length of cracks. Distribution of mixed-mode stress intensity factors (SIFs) and energy release rates (ERRs) along the fronts of cracks with different geometrical characteristics was investigated numerically, and the results can provide an interpretation for the experimental results. Based on numerical simulations of the propagation processes of single and two parallel cracks, the growth patterns and growth rates of 3-D cracks were studied. The simulation results of crack growth correspond well with the experimental phenomena.
\end{abstract}

Keywords: 3-D crack growth; Geometrical distribution of crack; Uniaxial tension; FRANC3D/BEM; Stress intensity factor $(S I F)$; Energy release rate $(E R R)$.

\footnotetext{
${ }^{1}$ Lecturer, Research Center of Geotechnical and Structural Engineering, Shandong Univ., Jinan 250061, P.R. China; formerly, Ph.D. Candidate, Graduate School of Science and Technology, Nagasaki Univ., Nagasaki 852-8521, Japan (corresponding author). E-mail: sdylei@gmail.com

2 Professor, Faculty of Engineering, Nagasaki Univ., Nagasaki 852-8521, Japan. E-mail: jiang@nagasaki-u.ac.jp

${ }^{3}$ Professor, Research Center of Geotechnical and Structural Engineering, Shandong Univ., Jinan 250061, P.R. China. E-mail: lishucai@sdu.edu.cn

${ }^{4}$ Assistant Professor, Faculty of Engineering, Nagasaki Univ., Nagasaki 852-8521, Japan. E-mail: libo@nagasaki-u.ac.jp
} 


\section{Introduction}

Cracks ubiquitously exist in rock masses, and play an important role in the strength and failure behavior of rock masses. As external loading increases, cracks grow and join with other neighboring ones, inducing stress redistribution and localized stress concentration, which subsequently lead to the failure of rock masses (Lajtai 1974). Therefore, the mechanism of crack growth and coalescence in rock masses remains as a fundamental and promising problem in rock mechanics and engineering practices.

To date, extensive studies have been carried out to investigate the growth of two-dimensional (2-D) cracks (plates with through cracks) in brittle materials under compression (e.g., Brace and Bombolakis 1963; Lajtai 1974; Ashby and Hallam 1986; Bobet and Einstein 1998; Bobet 2000). Since most cracks in nature are three-dimensional (3-D internal or surface cracks), recent research has attempted to model 3-D crack growth, which may provide a better interpretation for the rock failure behavior (e.g., Dyskin et al. 1994, 1999, 2003; Wong et al. 2004, 2008). Those studies have found that the propagation of 3-D cracks occurs mainly with the extension of wrapping wing cracks in the direction of maximum compressive stress, and with lateral extension growing in the depth direction of sample, making it much more complex than 2-D crack cases. Those studies have also shown that the geometrical characteristics of 3-D cracks, such as crack shape, crack location and the crack dip angle have significant influences on the crack growth.

Comparing with the cases under compression, the crack growth in rocks subjected to tension is more likely to cause the catastrophic failure without warning due to the fact that rock usually has a very low tensile strength. A number of experimental and theoretical studies based on the 2-D crack model under tension have been performed to predict the critical stress of crack growth and the fracture angle (Palaniswamy and Knauss 1972; Wu et al. 1977; Wu and Chang 1978; Sih 1991; Hsien-Yang and Chang 1995). Those studies provided a fundamental understanding of 2-D crack growth in brittle solids under tension, whereas, the growth mechanism of 3-D cracks in rocks under tension has received little attention and rare related works have been reported. In former studies, we have conducted uniaxial tension experiments on mortar samples containing single or two parallel 3-D internal cracks to investigate the effects of crack dip angle and spacing on sample strength (Li et al. 2009; Yang et al. 2010a and 2011). We proposed the growth patterns of internal cracks under tension through observing 
the crack growth trace on the failure sections of samples. However, since the mortar material is non-transparent, we could not capture the initiation and propagation processes of cracks and their 3-D growth information through direct observation.

The numerical simulation provides an effective way to study the crack growth process under various stress conditions and to capture the spatial fracture development. In recent years, the boundary element method (BEM) has been well developed and used as a powerful alternative to the finite element method (FEM) particularly in the analysis of 3-D fracture problems, due to its inherent advantage of high efficiency. Mi and Aliabadi (1994) adopted the dual BEM to study the 3-D crack growth by using an incremental crack-extension analysis. Singh et al. (1998) proposed the formulation of universal crack closure integral to evaluate the energy release rates (ERRs) and the stress intensity factors $(S I F \mathrm{~s})$ around 3-D crack fronts. Cornell Fracture Group has developed the BEM code of FRANC3D, which can automatically simulate the propagation of 3-D cracks and has been widely applied in the fracture analysis (e.g., Rahman et al. 2000; Dong and De Peter 2002; Barlow and Chandra 2005).

At present, some aspects of the 3-D crack growth and coalescence in rocks under tension, such as the spatial growth pattern of cracks, the initiation and propagation processes of cracks, and the interaction between cracks as well as its effect on the crack coalescence, are still not well understood. These aspects control the strength and failure behavior of rock masses and therefore need to be investigated. To study the growth and calescence of 3-D cracks, the effects of the geometrical characteristics of cracks (e.g., crack dip angle, crack spacing and crack intensity) should be taken into account. The crack dip angle affects the stress distribution around cracks and the growth pattern of cracks. The crack spacing affects the crack interaction and the coalescence of cracks, and the crack intensity has an obvious influence on the mechanical properties of a fractured rock.

In this study, we briefly summarize the results of uniaxial tension experiments obtained before, including the effects of crack dip angle, spacing and intensity on the strengths and failure modes of mortar samples. Since the growth process of internal cracks could not be observed directly in the non-transparent mortar samples during experiments, as a supplement, the corresponding numerical simulations were performed using FRANC3D. In numerical studies, the distribution of mixed-mode SIFs and ERRs along the fronts of cracks with varying dip angles and spacing was studied, and the propagation and coalescence processes of 3-D cracks were 
numerically simulated. The numerical studies can serve as an effective complement to experimental studies, providing more insights into the growth and coalescence mechanisms of 3-D cracks under tension, such as the initiation position, the spatial growth pattern, as well as the effects of cracks' geometrical characteristics on their growth and coalescence.

\section{Experiments and Discussions}

\section{Preparation of Experiment}

In the experiments, a new type of rock-like mortar material (a mixture of Portland cement, fine sand and water with the mixing ratio by weight of $1: 2.34: 0.35$ ) was developed to simulate sandstone. Mechanical properties of the mortar material were measured through unconfined compression and tension tests and the three-point bending test. As shown in Table 1, this mortar material exhibits similar properties to the typical sandstone (Farmer 1983; Atkinson and Meredith 1987).

Table 1. Physico-mechanical Properties of Mortar and Sandstone

\begin{tabular}{cccc}
\hline Parameter & Index & Mortar & Sandstone \\
\hline Density & $\rho\left(\mathrm{g} / \mathrm{cm}^{3}\right)$ & 2.3 & $2.1 \sim 2.4$ \\
Compressive strength & $\sigma_{\mathrm{c}}(\mathrm{MPa})$ & 35.5 & $20 \sim 170$ \\
Tensile strength & $\sigma_{t}(\mathrm{MPa})$ & 2.8 & $4 \sim 25$ \\
Modulus of elasticity & $E(\mathrm{Gpa})$ & 17.9 & $3 \sim 35$ \\
Poisson's ratio & $v$ & 0.19 & $0.02 \sim 0.20$ \\
Fracture toughness & $K_{\mathrm{IC}}\left(\mathrm{MPa} \cdot \mathrm{m}^{0.5}\right)$ & 0.51 & $0.22 \sim 2.66$ \\
\hline
\end{tabular}
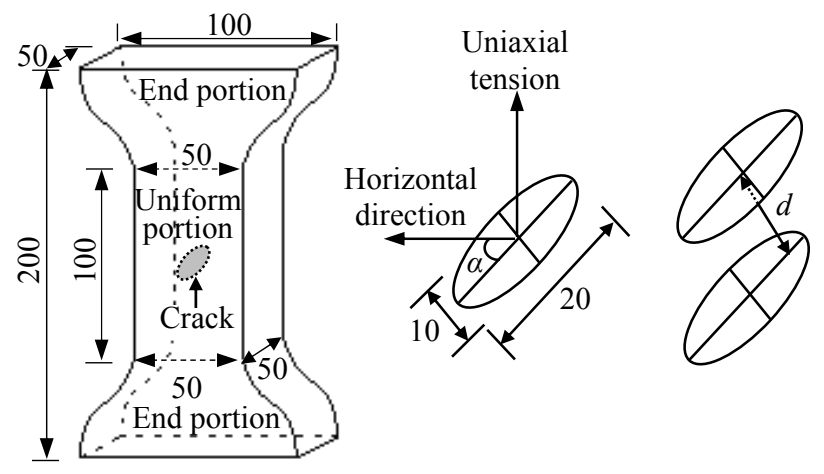
(a) Sample
(b) A single crack
(c) Two cracks

Fig. 1. Shapes and dimensions of artificial sample and elliptical internal cracks (Unit: $\mathrm{mm})$ 
Shapes and dimensions of the artificial sample and internal crack are shown in Fig. 1. The sample has a bone-like shape to reduce the stress concentration when applying tensile stresses on the upper and lower boundaries. The internal cracks were created by embedding elliptical polyester films with a thickness of $0.25 \mathrm{~mm}$ into the geometric centers of samples during the casting process. Polyester films were greased and held within samples by cotton threads to model non-frictional open cracks.

Three sets of samples were processed to investigate the influences of crack dip angle, spacing and intensity on sample strengths and failure modes, respectively. Set-1 samples contain single cracks with varying dip angles $\alpha$ (inclination to the horizontal direction, Fig. 1b), ranging from $0^{\circ}$ to $60^{\circ}$ with an increment of $15^{\circ}$. Each sample in Set-2 contains two vertically aligned parallel cracks with an inclination of $45^{\circ}$ (Fig. 1c). The spacing $d$ between two cracks was set as $0,5,10,15,20$ and $25 \mathrm{~mm}$, respectively, so that the dimensionless spacing factor $d / 2 a$ ranges from 0 to 1.25 ( $2 a$ is the length of crack long axis). Set-3 samples contain different number of vertically aligned parallel cracks (the crack intensity ranges from 1 to 3 , corresponding to the crack number), with the crack inclination of $45^{\circ}$ and the crack spacing of $5 \mathrm{~mm}$.

Samples were uniaxially loaded using a $3000 \mathrm{kN}$ servo-controlled rigid testing device in a velocity controlled mode at a rate of $0.002 \mathrm{~mm} / \mathrm{s}$. To assure that the tensile stress passes through the center line of sample during tests, a locating apparatus for bonding tension tests was utilized, which can rotate freely along the axial direction of sample and slide in the lateral direction (Yang et al. 2010a). This locating apparatus can adjust the axial direction of sample effectively, helping improve the accuracy of tension experiments.

\section{Experimental Results}

Uniaxial tensile stress-strain curves of the Set-1 samples and the intact sample are shown in Fig. 2. At the beginning of loading, the curve is nonlinear due to the opening of pre-existing crack and microcracks in the sample. After that, the curve exhibits a large linear portion, corresponding to the elastic-deformation stage of sample (the onset of this stage has been indicated on each curve). It can be found that the load-deformation process of the fractured sample is primarily occupied by the large elastic-deformation stage, which produces more than $50 \%$ of the total deformation. After the external stress reaches the tensile strength of sample, sample fractures 
abruptly with a quick drop of stress. The deformation and failure behavior of rock-like material under tension is quite different from that under compression (Yang et al. $2010 \mathrm{~b}$ ). The existence of the pre-existing crack results in the decrease of sample strength $\sigma_{\mathrm{p}}$, which is also affected by the crack dip angle $\alpha$ ( $\sigma_{\mathrm{p}}$ decreases with the decrease of $\alpha$ ). As $\alpha$ decreases, the stress component normal to the initial crack plane increases, therefore the mode-I (opening) fracture is more likely to occur, and the sample strength decreases. The ultimate strains of samples do not follow a certain law, which may be due to the different geometrical distributions of pre-existing cracks and the material's heterogeneity.

The instantaneous fracture of the Set-1 samples occurred in the middle of the samples and the failure sections were approximately perpendicular to the external stress. The growth trace of internal cracks can be observed on the failure sections of samples. In the case of a horizontal crack $\left(\alpha=0^{\circ}\right)$, only mode-I (opening) fracture occurred and caused the brittle failure of the sample (Fig. 3a). The wing cracks and

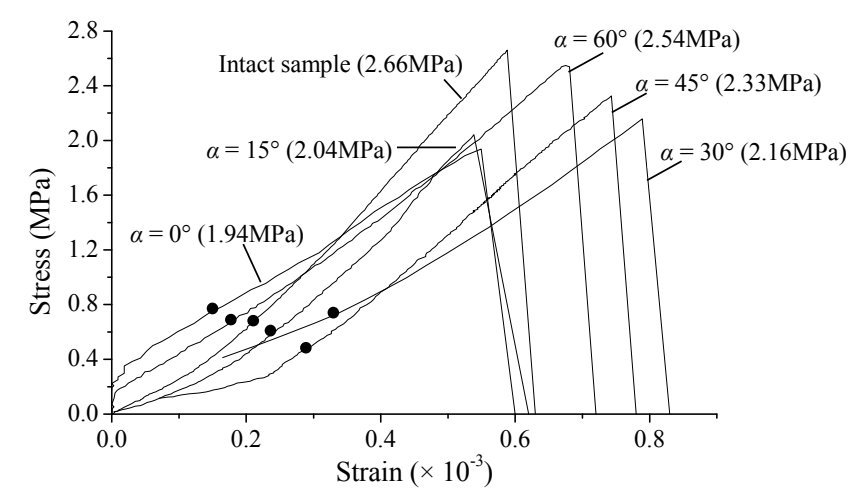

Fig. 2. Uniaxial tensile stress-strain curves of the Set-1 samples
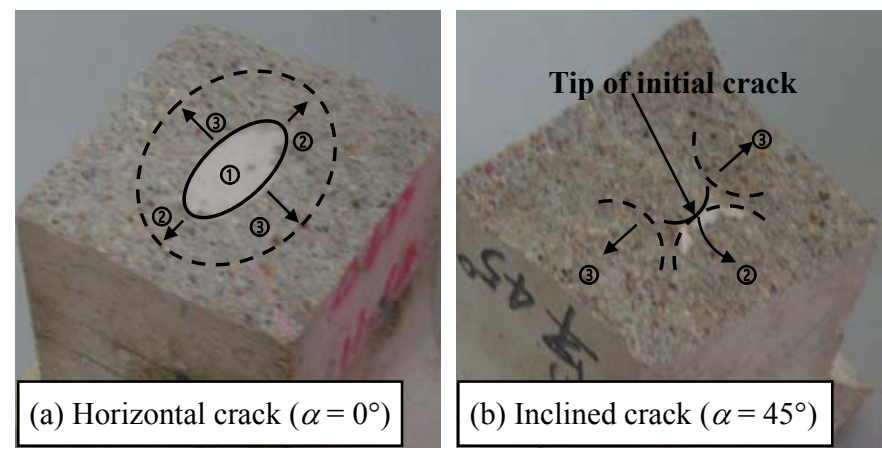

Fig. 3. Failure sections of samples with single cracks (1) : Initial crack; (2) : Wing crack; (3) : Lateral extension) 
the lateral extension initiated from the tips of the crack long and short axes, respectively, which propagated along the initial crack plane with the inclination and propagation angles to the initial crack plane approximately equal to $0^{\circ}$. In the cases of inclined cracks $\left(15^{\circ} \leq \alpha \leq 60^{\circ}\right)$, the propagation trace of wing crack can be confirmed on the failure sections of samples, indicating that the newly-generated wing crack led to the sample failure (Fig. $3 \mathrm{~b}$, e.g. $\alpha=45^{\circ}$ ). The initiation of the wing crack was approximately perpendicular to the initial crack plane. During the crack growth process, the propagation of wing crack gradually changed to the horizontal direction, with the propagation angle progressively approaching to the dip angle of initial crack.

The tensile stress-strain curves of the Set-2 samples with two parallel cracks are shown in Fig. 4, indicating that the sample strength varies with the crack spacing. When $d / 2 a$ ranges from 0 to $1, \sigma_{\mathrm{p}}$ decreases as the crack spacing increases due to the increase of the weak rock bridge zone between cracks, where the stress fields resulted from the two cracks are strongly superposed, causing the rapid propagation and

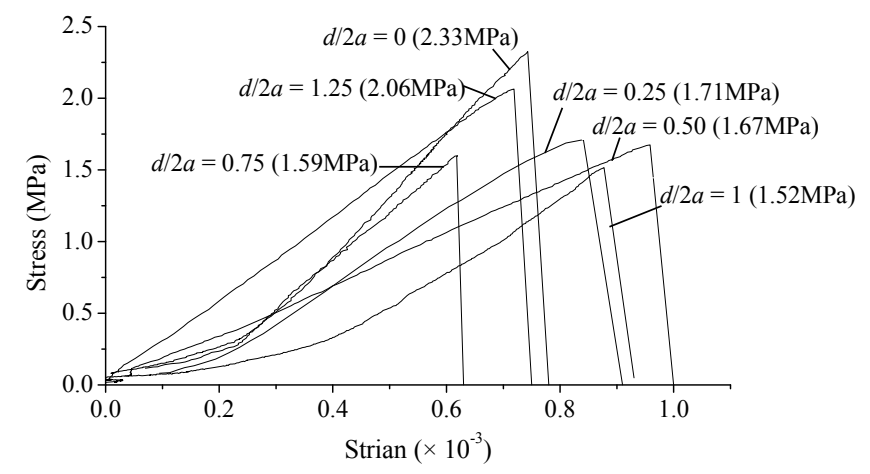

Fig. 4. Uniaxial tensile stress-strain curves of the Set-2 samples
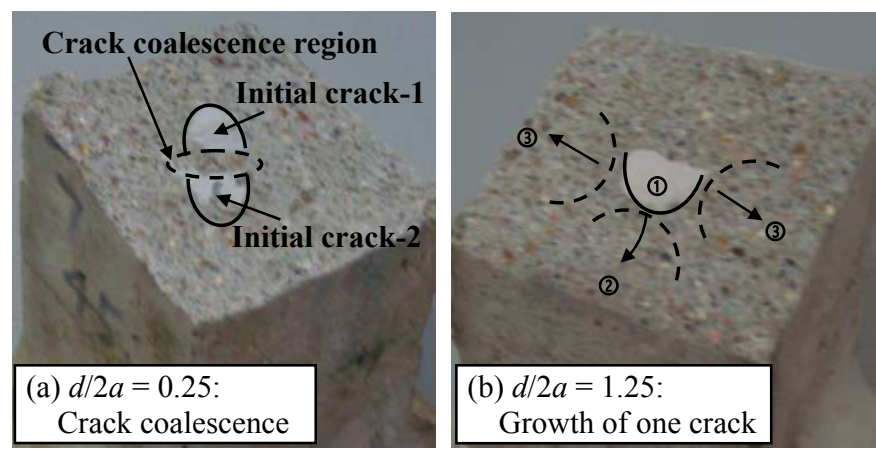

Fig. 5. Failure sections of samples with two cracks (1) : Initial crack; (2): Wing crack; (3) : Lateral extension) 


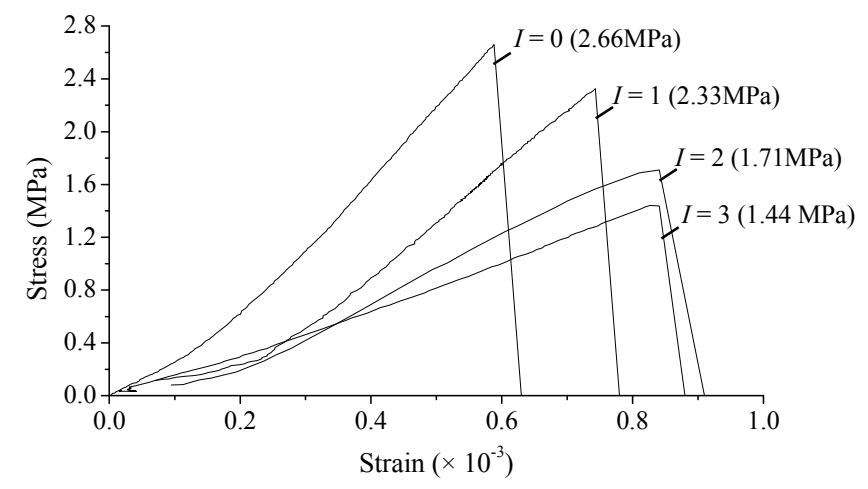

Fig. 6. Uniaxial tensile stress-strain curves of the Set-3 samples

coalescence of initial cracks. Then $\sigma_{\mathrm{p}}$ increases after $d / 2 a$ exceeds 1.0 , due to that the interaction effect between the two cracks gradually reduces with spacing.

Failure of the Set-2 samples mainly resulted from the growth and coalescence of initial cracks. In the range of small crack spacing $(d / 2 a \leq 1)$, the interaction between cracks was strong and the two cracks propagated and joined with each other, leading to the sample failure (Fig. 5a, e.g. $d / 2 a=0.25$ ). In the case that the crack spacing was large $(d / 2 a>1)$, two cracks grew separately, and the one that grew more sufficiently due to the material's heterogeneity, caused the sample failure (Fig. 5b).

Set-3 samples were tested to investigate the effect of crack intensity $I$. The stress-strain curves shown in Fig. 6 indicate that the sample strength and elastic modulus decrease with the increase of crack intensity. Since the crack spacing of Set-3 samples is small $(d / 2 a=0.25)$, initial cracks grew and joined with adjacent ones, leading to the sample failure, similarly to the two-crack cases (Fig. 5a).

\section{Method and Models of Numerical Simulations}

\section{Brief Description of FRANC3D/BEM}

Boundary element method (BEM) is a numerical method of solving the boundary integral equation that is mathematically equivalent to the original partial differential equation. The most significant feature of BEM is the reduction of the dimensionality of a problem, as it only requires the discretization of surfaces rather than the domain, which improves the calculating efficiency to a great extent (Rizzo 1967).

The BEM code of FRANC3D/Classic Version 3.2 is a specialized and powerful fracture analysis program, capable of accurately evaluating SIFs along 3-D crack 
fronts and representing the propagation of these cracks (Carter et al. 2000; Cornell Fracture Group 2003). FRANC3D calculates SIFs by using a displacement correlation technique based on the interpolated values calculated at points with measured distances from the geometric crack fronts. The displacements on the crack surface are directly related to three modes of SIFs in forms of Eqs. (1)-(3), where COD, CSD and $C T D$ represent the opening, sliding and tearing displacements of a crack respectively; $K_{\mathrm{I}}, K_{\mathrm{II}}$ and $K_{\mathrm{III}}$ represent the $S I F \mathrm{~S}$ for mode I, II and III fracture respectively; $r$ denotes the distance from a calculating point to the crack front; $E$ and $v$ are Young's modulus and Poisson's ratio respectively (Sousa et al. 1989). After the displacements of points around the crack front are obtained, the $S I F$ s can be calculated.

$$
\begin{aligned}
& C O D=4 K_{\mathrm{I}}\left(1-v^{2}\right)(2 r / \pi)^{1 / 2} / E \\
& C S D=4 K_{\mathrm{II}}\left(1-v^{2}\right)(2 r / \pi)^{1 / 2} / E \\
& C T D=4 K_{\mathrm{III}}(1+v)(2 r / \pi)^{1 / 2} / E
\end{aligned}
$$

The crack propagation is controlled by the relative magnitudes of SIFs at the crack front. $K_{\mathrm{I}}$ is related to the opening of the crack and causes the in-plane growth of the crack. $K_{\text {II }}$ produces in-plane sliding and tilting of the crack front, while $K_{\text {III }}$ represents out-of-plane tearing and produces twisting of the crack front. The propagation direction of the complex 3-D crack can be determined by using some fracture theories (e.g., the theory of maximum tangential stress, the theory of maximum strain energy release rate and the theory of minimum strain energy density) integrated in the FRANC3D framework. In this study, the theory of maximum strain energy release rate was utilized, in the form of Eq. (4).

$$
E R R=\left(K_{\mathrm{I}}^{2}+K_{\mathrm{II}}^{2}\right)\left(1-v^{2}\right) / E+K_{\mathrm{III}}^{2}(1+v) / E
$$

The amount of crack extension at a certain point $\left(\Delta l_{\mathrm{i}}\right)$ was determined through the power-law relationship between $S I F$ s and the crack growth length, as defined by:

$$
\Delta l_{i}=\Delta l_{\max }\left(K_{\mathrm{I}}-K_{\mathrm{IC}}\right) /\left(K_{\mathrm{I} \max }-K_{\mathrm{IC}}\right)
$$

where $K_{\text {Imax }}$ is the maximum value of $K_{\mathrm{I}}$ along the crack front; $K_{\mathrm{IC}}$ is the fracture toughness of the material; $\Delta l_{\max }$ denotes the maximum length of crack extension for the position of $K_{\text {Imax. }}$

\section{Numerical models}

The uniform-section segment of the experimental sample with the same size and 

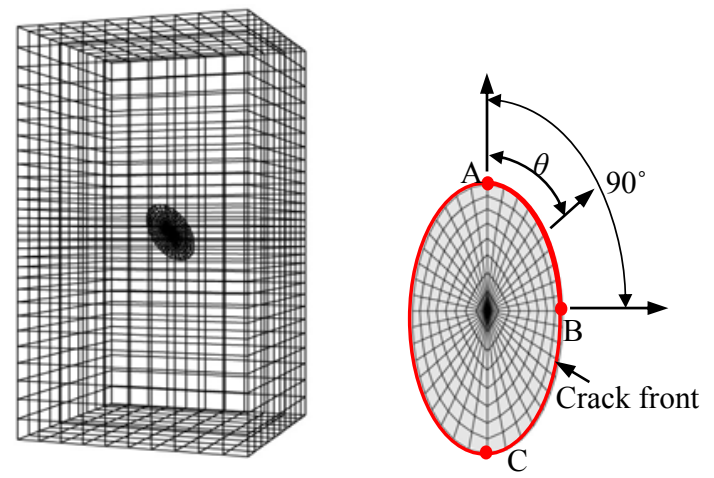

Fig. 7. Meshes of the numerical model and crack (a single crack)

physico-mechanical properties was used in the numerical models. Corresponding to the laboratory test procedure, two sets of numerical models were established to investigate the effects of crack dip angle and spacing on the crack growth respectively. In these models, the size and arrangement of cracks were identical to those in experimental samples. Elliptical cracks were created by defining the coordinates of their geometric centers, the crack inclinations, and the dimensions of cracks. The open crack was topologically represented by a pair of faces, which use the same front edges and vertices and form a null volume region with zero strength. Those faces, edges and vertices can be torn apart to simulate the crack propagation if the external stress is large enough (Carter et al. 2000). The meshes on the model surface and crack surface are shown in Fig. 7. Meshes on the model surface are fine near the internal crack and coarse near the upper and lower ends of the model, with a total number of 1400 . On each crack surface, 320 4-sided mapped meshes were generated to calculate the SIFs and ERRs of cracks.

The uniaxial tensile stress $\sigma$ was imposed on the upper boundary of model, and the axial displacement on the lower boundary was set to be zero. To investigate the distribution of mixed-mode $S I F \mathrm{~s}$ and ERRs, a position angle $\theta$ was defined as the angle from the crack long axis to the radial line corresponding to one particular point, which was used to mark the point position along the crack front (Fig. 7).

The normalization of mixed-mode $\operatorname{SIF\mathrm {s}}\left(K_{\text {Inor }}, K_{\text {IInor }}\right.$ and $\left.K_{\text {IIInor }}\right)$ and ERRs $\left(E R R_{n o r}\right)$ along the fronts of all cracks was performed by using Eqs. (6)-(8) (Ayhan 2007), where $a$ and $b$ represent the semi-lengths of long and short axes of the elliptical crack respectively; $\sigma$ is the uniaxial tensile stress; and $v$ is the Poisson's ratio of material. 


$$
\begin{gathered}
K_{\mathrm{Inor}}=K_{\mathrm{I}} / K_{R}, \quad K_{\mathrm{IInor}}=K_{\mathrm{II}} / K_{R}, \quad K_{\mathrm{IIInor}}=K_{\mathrm{III}} / K_{R} \\
K_{R}=\sigma\left\{\pi a /\left[1+1.464(a / b)^{1.65}\right]\right\}^{1 / 2} \\
E R R_{\text {nor }}=\left(K_{\mathrm{Inor}}{ }^{2}+K_{\mathrm{II} n o r}{ }^{2}\right)\left(1-v^{2}\right)+K_{\mathrm{IIInor}}{ }^{2}(1+v)
\end{gathered}
$$

\section{Numerical Results and Discussions}

\section{Case of Single Cracks}

\section{Distribution of SIFs and ERRs}

The normalized mixed-mode $S I F$ s along single crack fronts with varying dip angles $\alpha$ are shown in Fig. 8. Due to the symmetry, only SIFs along $1 / 4$ crack fronts (from the upper endpoint of the crack long axis to the right endpoint of the crack short axis, A-B in Fig. 7 and $0^{\circ} \leq \theta \leq 90^{\circ}$ ) are plotted. Fig. 8a indicates that $K_{\text {I }}$ increases continuously with the increasing $\theta$ from $0^{\circ}$ to $90^{\circ}$. The maximum and minimum values of $K_{\text {I }}$ are located at the endpoints of crack short and long axes respectively. At any position along the crack front, $K_{\mathrm{I}}$ increases with the decrease of $\alpha$ and reaches the maximum at $\alpha=0^{\circ}$ (the horizontal crack). Moreover, the increment of $K_{\mathrm{I}}$ from the minimum value to the maximum value for a crack becomes larger as $\alpha$ decreases.

As shown in Fig. $8 \mathrm{~b}, K_{\text {II }}$ decreases approximately in a linear manner with the increase of $\theta$ from $0^{\circ}$ to $90^{\circ}$. At the endpoint of the crack long axis, $K_{\text {II }}$ reaches its maximum, while at the endpoint of the crack short axis, $K_{\text {II }}$ decreases to its minimum (0). At any position, $K_{\text {II }}$ increases with the increase of $\alpha$ when $0^{\circ} \leq \alpha \leq 45^{\circ}$, but decreases after $\alpha$ exceeds $45^{\circ}$. Fig. $8 \mathrm{~b}$ also reveals that the values of $K_{\text {II }}$ when $\alpha=15^{\circ}$ and $30^{\circ}$ are approximately equal to those when $\alpha=75^{\circ}$ and $60^{\circ}$ respectively.

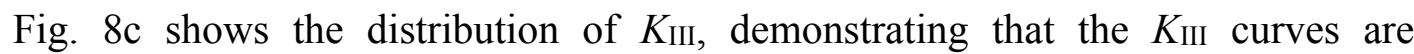
parabolic with the maximum values located at the endpoints of the crack short axes. At endpoints of the long axes, $K_{\text {III }}$ has its minimum value equaling to zero. The influence of $\alpha$ on the $K_{\text {III }}$ distribution is generally similar to that in the case of $K_{\text {III }} K_{\text {III }}$ reaches the maximum when $\alpha=45^{\circ}$, and $K_{\text {III }}$ increases as $\alpha$ increases from $0^{\circ}$ to $45^{\circ}$, but decreases after $\alpha$ exceeds $45^{\circ}$.

The total energy release rate $(E R R)$, which involves three modes of SIFs (Eq. 4), is commonly utilized to determine the safety/failure conditions and to predict the growth of 3-D cracks. The normalized ERRs along single crack fronts are calculated from Eq. 
(8) and are plotted in Fig. 9. It can be derived that the distribution of ERRs is similar to that of $K_{\mathrm{I}}$, implying that $K_{\mathrm{I}}$ makes a principal contribution to the $E R R$ distribution. The ERR along the crack front increases with the increase of $\theta$ and reaches its maximum when $\theta=90^{\circ}$, indicating that the growth of single cracks will initiate from the regions close to the endpoints of the crack short axes. The ERR increases continuously as $\alpha$ decreases, revealing that with the decrease of dip angle, the internal

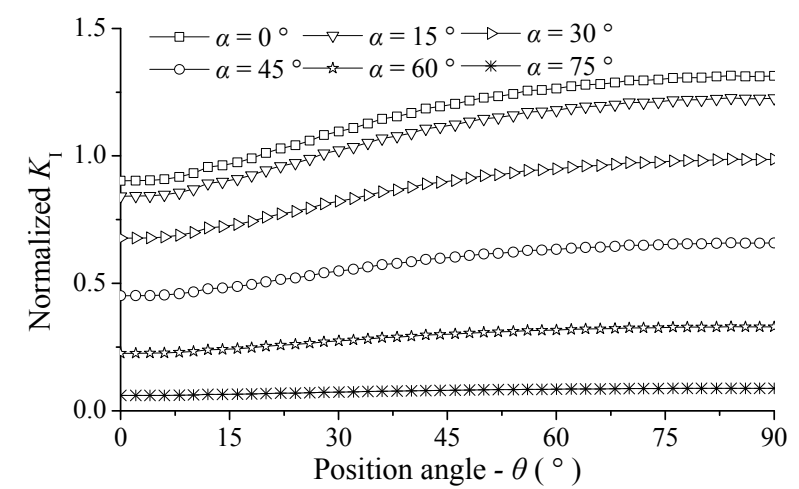

(a) Distribution of normalized $K_{\mathrm{I}}$

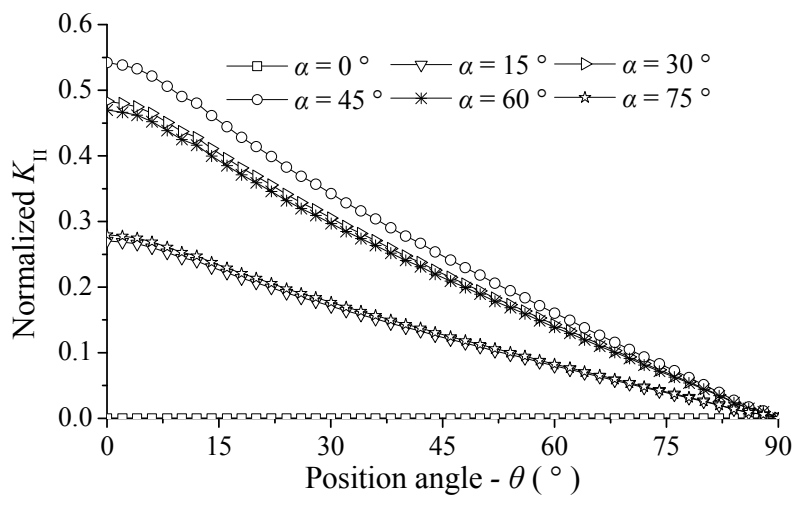

(b) Distribution of normalized $K_{\text {II }}$

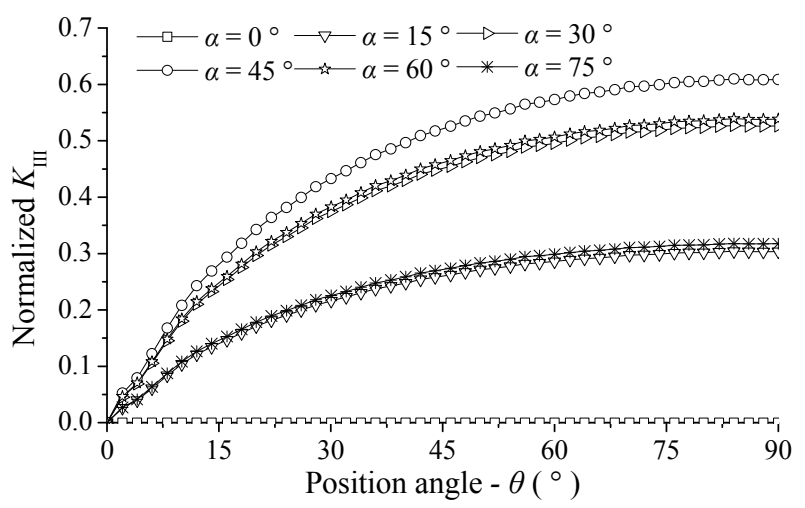

(c) Distribution of normalized $K_{\text {III }}$

Fig. 8. Distribution of normalized SIFs along single crack fronts 


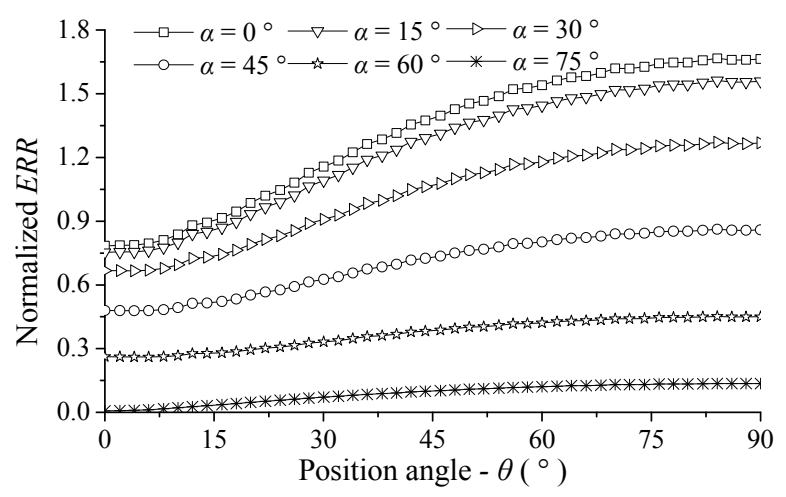

Fig. 9. Distribution of normalized ERRs along single crack fronts

crack is more easily to grow, leading to the fracture of samples and the decrease of sample strength. The numerical results of ERR distribution along single crack fronts provide an explanation for the effect of crack dip angle on the sample strength (Fig. 2).

\section{Propagation of Single Cracks}

The tensile propagation processes of single cracks were investigated by using FRANC3D, where 4 steps of crack propagation were simulated for each case until the total growth size of each crack exceeded its initial size (Fig. 10). Simulation results indicate that the propagation patterns of single cracks fall into two categories according to the crack dip angle, including the pattern of horizontal crack $\left(\alpha=0^{\circ}\right)$ and the pattern of inclined cracks (e.g. $\alpha=45^{\circ}$ ).

For the horizontal crack (Fig. 10a), only mode-I (opening) fracture occurs due to the uniaxial tensile stress $\left(K_{\mathrm{I}} \neq 0, K_{\mathrm{II}}, K_{\mathrm{III}}=0\right)$, in the form of crack extension along its initial plane. The horizontal crack starts its lateral extension from the endpoint of the crack short axis (point B), and then generates the wing crack from the endpoint of the long axis (point A). To investigate the growth of crack quantitatively, the growth

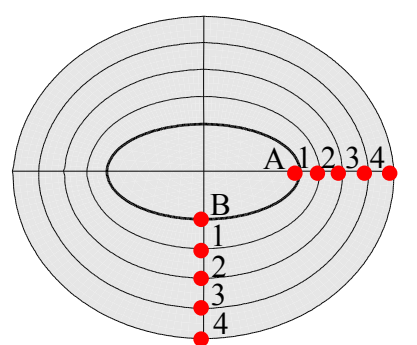

(a) Horizontal crack $\left(\alpha=0^{\circ}\right)$

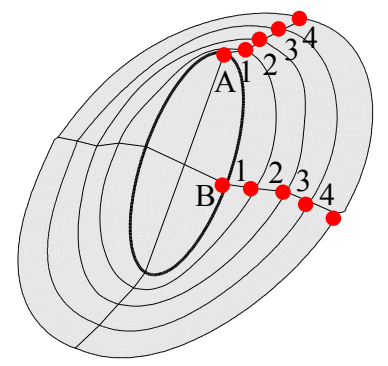

(b) Inclined $\operatorname{crack}\left(\alpha=45^{\circ}\right)$

Fig. 10. Propagation processes of single cracks under tension 
Table 2. Growth Lengths of Single Cracks at the Endpoints of Long and Short Axes in Every Calculating Step

\begin{tabular}{c|ccr|ccr}
\hline \multirow{2}{*}{ No. of steps } & \multicolumn{3}{|c|}{$\begin{array}{c}\text { Horizontal crack } \\
\left(\alpha=0^{\circ}\right)\end{array}$} & \multicolumn{3}{c}{$\begin{array}{c}\text { Inclined crack } \\
\left(\text { e.g. } \alpha=45^{\circ}\right)\end{array}$} \\
\cline { 2 - 7 } & $L_{\mathrm{A}}(\mathrm{mm})$ & $L_{\mathrm{B}}(\mathrm{mm})$ & $L_{\mathrm{A}} / L_{\mathrm{B}}$ & $L_{\mathrm{A}}(\mathrm{mm})$ & $L_{\mathrm{B}}(\mathrm{mm})$ & $L_{\mathrm{A}} / L_{\mathrm{B}}$ \\
\hline 1 & 2.06 & 3.00 & 0.69 & 2.06 & 3.03 & 0.68 \\
2 & 2.43 & 3.00 & 0.81 & 2.25 & 2.61 & 0.86 \\
3 & 2.59 & 2.98 & 0.87 & 2.44 & 2.31 & 1.06 \\
4 & 2.72 & 2.98 & 0.91 & 2.86 & 2.20 & 1.30 \\
\hline
\end{tabular}

lengths at points $\mathrm{A}$ and $\mathrm{B}$ (denoted as $L_{\mathrm{A}}$ and $L_{\mathrm{B}}$ ) were measured during every step, as listed in Table 2. The results demonstrate that the lateral extension $\left(L_{\mathrm{B}}\right)$ is much faster than the growth of wing crack $\left(L_{\mathrm{A}}\right)$ at the beginning, but the growth length per step (growth rate) of wing crack increases gradually during the crack propagation process with the increase of the growth ratio defined by $L_{\mathrm{A}} / L_{\mathrm{B}}\left(0.69<L_{\mathrm{A}} / L_{\mathrm{B}}<1\right)$. Then the elliptical crack grows gradually into a circular shape almost with the same growth rate at points $\mathrm{A}$ and $\mathrm{B}$, and the in-plane growth of crack finally leads to the fracture of sample. It can be found that the numerical result agrees well with the failure phenomenon observed in laboratory experiments (Fig. 3a).

In the cases of inclined cracks (Fig. 10b), the wrapping wing crack and lateral extension are the major growth patterns. Similar to the horizontal crack, the lateral extension firstly initiates from the front of crack short axis, and grows along the initial crack plane. Then the wrapping wing crack develops from the front of crack long axis, propagating initially in the direction approximately perpendicular to the crack plane and then changing to the horizontal direction. Table 2 also lists the growth lengths of inclined crack at the endpoints of long and short axes during every step, indicating that at the beginning the lateral extension grows more quickly than the wing crack. During the propagation process, the growth rate of lateral extension decreases gradually, while the growth rate of wing crack increases. After the lateral extension exceeds the half-length of crack short axis, the growth rate of wing crack surpasses that of the lateral extension, with the ratio $L_{\mathrm{A}} / L_{\mathrm{B}}>1$. The combined propagation of wrapping wing crack and lateral extension forms a spatially-distorted plane, which causes the overall fracture. The rapid growth of wing crack breaks the sample and the growth trace of wing crack can be observed on the sample failure section (Fig. 3b). 


\section{Case of Two Parallel Cracks}

\section{Distribution of SIFs and ERRs}

In the models that contain two parallel cracks, the interaction between adjacent cracks brings obvious influences on the distribution of $S I F \mathrm{~s}$ and $E R R \mathrm{~s}$ along crack fronts as well as on the growth pattern of cracks. Due to the symmetry, the normalized SIFs along the upper crack fronts between endpoints of the crack long axis (A-C in Fig. 7, $\left.0^{\circ} \leq \theta \leq 180^{\circ}\right)$ in all cases with varying crack spacing are plotted in Fig. 11.

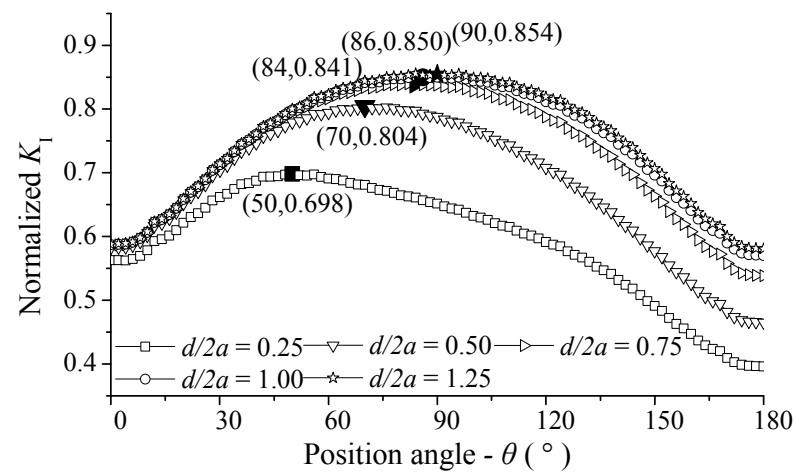

(a) Distribution of normalized $K_{\mathrm{I}}$

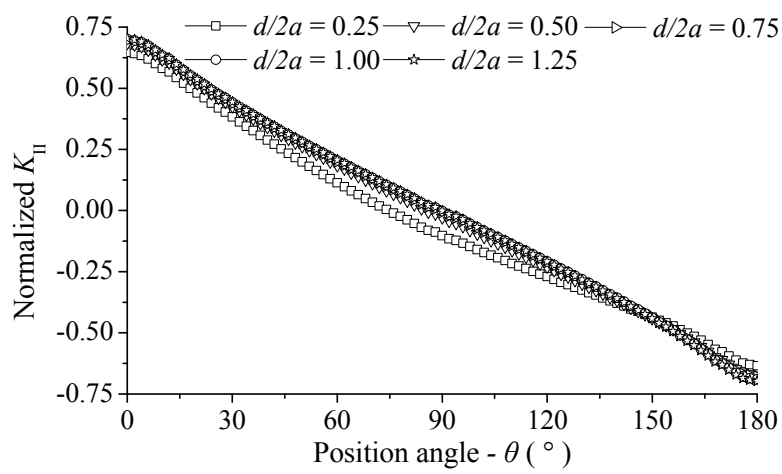

(b) Distribution of normalized $K_{\text {II }}$

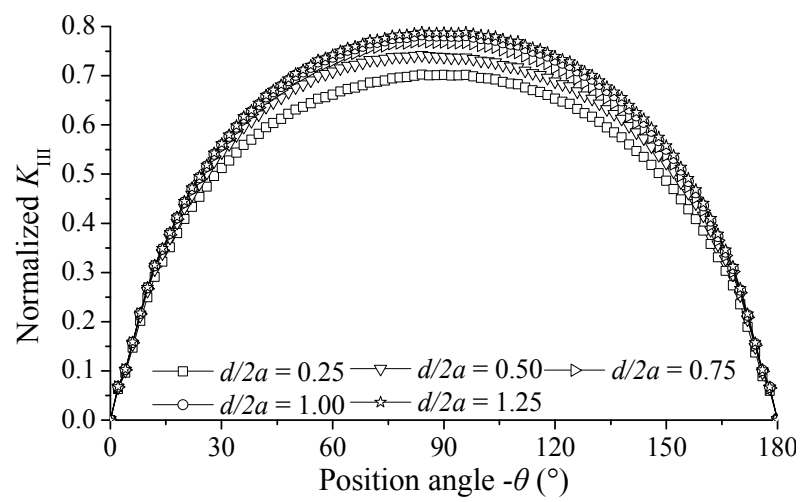

(c) Distribution of normalized $K_{\text {III }}$

Fig. 11. Distribution of normalized SIFs along the upper crack front of two parallel cracks 


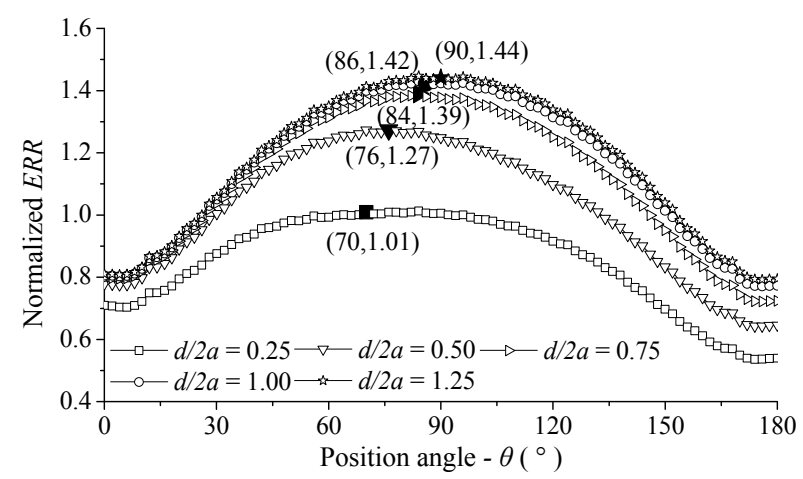

Fig. 12. Distribution of normalized ERRs along the upper crack front of two parallel cracks

Fig. 11a shows the distribution of normalized $K_{\mathrm{I}}$, exhibiting a different distributing pattern from the situation of single cracks due to the crack interaction. In the crack spacing range of this study $(0.25 \leq d / 2 a \leq 1.25)$, the maximum values of $K_{\text {I }}$ appear at the angles $\theta$ ranging from $50^{\circ}$ to $90^{\circ}$ (as marked in Fig. 11a), while the minimum values of $K_{\mathrm{I}}$ occur at the lower endpoints of the long axes. $K_{\mathrm{I}}$ along the crack front increases gradually as $d / 2 a$ increases and reaches the maximum when $d / 2 a=1.25$. After that, $K_{\mathrm{I}}$ decreases with the increase of crack spacing. Moreover, the crack spacing also affects the position where the maximum $K_{\mathrm{I}}$ appears. When the crack spacing is small, the maximum $K_{\text {I }}$ occurs approximately at the middle position of $1 / 4$ crack front, and as the crack spacing increases, the position of the maximum $K_{\text {I moves }}$ continuously to the endpoint of short axis, just similar to the situation of single cracks.

The normalized $K_{\text {II }}$ of two-crack cases has a similar distributing pattern (a linear manner along crack fronts) to the single-crack cases (Fig. 11b), with its maximum and minimum values (absolute values) located at the endpoints of long and short axes of cracks respectively. Numerical results also reveal that the crack spacing has a weak influence on the $K_{\text {II }}$ values and all the curves in Fig. $11 \mathrm{~b}$ are close to each other.

As shown in Fig. 11c, the normalized $K_{\text {III }}$ of two-crack cases distributes along the crack front in a similar manner to that of the single-crack cases. The values of $K_{\text {III }}$ increase with the increase of crack spacing in the range of $0.25 \leq d / 2 a \leq 1.25$, and after that $K_{\text {III }}$ decreases as the spacing increases.

The distributing pattern of normalized ERRs along the upper crack is principally governed by the $K_{\mathrm{I}}$ distribution (Fig. 12). The maximum values of these $E R R$ curves are located in the angle range of $70^{\circ} \leq \theta \leq 90^{\circ}$, indicating that cracks start propagating 
from locations near the endpoints of the short axes. Since the average ERR on the upper half of crack front $\left(0 \leq \theta \leq 90^{\circ}\right)$ is larger than that on the lower half $\left(90^{\circ} \leq \theta \leq\right.$ $180^{\circ}$ ), the crack grows more sufficiently from the upper half front than from the lower half. With the increase of $d / 2 a$, the value of $E R R$ increases and reaches its maximum when $d / 2 a=1.25$. Then $E R R$ decreases after $d / 2 a$ exceeds 1.25 , due to the weakening of crack interaction. The influence of crack spacing on $E R R$ values corresponds to the experimental results of spacing's effect on the sample strength to some extent (Fig. 4). As the $E R R$ increases with crack spacing until it reaches a critical value $(d / 2 a=1.25$ in numerical simulations and $d / 2 a=1$ in experiments), cracks in numerical models and laboratory samples are more easily to propagate, leading to the decrease of sample strength. After the crack spacing exceeds the critical value, the interaction between cracks becomes weak and cracks propagate separately in the pattern similar to the situation of samples with single cracks, leading to the increase of sample strength. However, the critical values are slightly different between numerical and experimental results, which may be caused by the heterogeneity of the tested material.

\section{Propagation of Two Parallel Cracks}

The tensile propagation pattern of two parallel cracks is similar to that of a single crack, in the form of lateral extension and growth of wing cracks (Fig. 13, e.g. $d / 2 a=$ 0.25). However, due to the interaction between cracks, the initiation position and growth rate of initial cracks are different. The propagation of two cracks initiates from the positions at a short distance from the endpoints of short axis $\left(\theta=70^{\circ}\right.$ at the upper

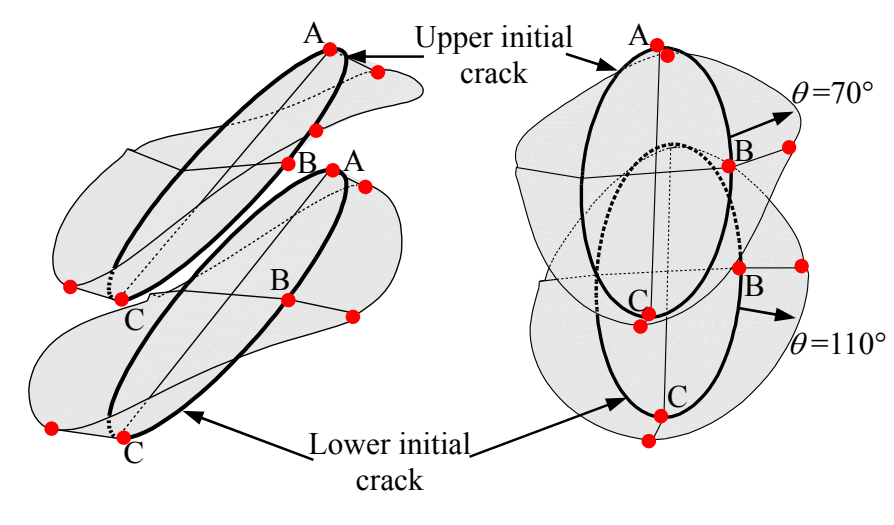

(a) The lateral view

(b) The front view

Fig. 13. Propagation of two parallel cracks $(d / 2 a=0.25)$ 
crack and $\theta=100^{\circ}$ at the lower crack, as marked in Fig. 13b). According to the distribution of ERRs (Fig. 12), it can be found that with the increase of crack spacing, the initiation positions move gradually to the endpoints of crack short axis. The simulation results indicate that the growth rate of the upper (lower) half of the upper (lower) crack is larger than that of the lower (upper) half, showing that the wrapping wing cracks in the region between two initial cracks (rock bridge zone) grow more sufficiently than those growing out of the rock bridge zone. With the propagation of wing cracks, the rock bridge zone is continuously cut and finally the fracture occurs throughout the rock bridge zone. This propagation process of two cracks agrees well with the experimental observation (Fig. 5a), where the coalescence regions connecting two initial cracks can be clearly observed.

After crack spacing exceeds the critical value $(d / 2 a=1.25$ in numerical simulations and $d / 2 a=1$ in experiments), the interaction between cracks gets weaker and those two cracks propagate separately in the manner similar to single cracks. Finally, the one that grows more sufficiently due to the material's heterogeneity will lead to the fracture of the sample. Therefore, only the propagation trace of one crack can be observed on the failure section of the sample (Fig. 5b).

\section{Conclusions and Discussions}

In this study, uniaxial tension experiments on mortar samples containing 3-D internal cracks and their corresponding numerical simulations by using the BEM code of FRANC3D were carried out to investigate the growth and coalescence of 3-D cracks in the rock-like material under tension. The mechanical and failure properties of different samples were obtained in experiments, and the mixed-mode SIFs and ERRs along crack fronts as well as the crack growth process were investigated in numerical studies to provide more information on the 3-D crack growth.

The mechanical and failure behavior of samples is significantly affected by the geometrical characteristics of cracks. The sample strength decreases with the decrease of crack dip angle and the increase of crack intensity. In the case of single cracks, the sample failure resulted from the in-plane growth of horizontal crack or the growth of wing cracks generated at inclined crack tips. For samples with two parallel cracks, the sample strength decreases with the increase of crack spacing when $0 \leq d / 2 a \leq 1$, and then increases after $d / 2 a$ exceeds 1 . At small spacing $(d / 2 a \leq 1)$, two cracks grew and 
joined together, leading to the sample failure, while at large spacing $(d / 2 a>1)$, the crack that grew more sufficiently caused the sample failure.

The distribution of mixed-mode $S I F \mathrm{~s}$ and ERRs along the crack fronts controls the propagation pattern of 3-D cracks. Numerical results on samples with single cracks indicate that $K_{\mathrm{I}}, K_{\mathrm{II}}$ and $K_{\mathrm{III}}$ have different distributing patterns along the crack front and these values are also affected by the crack dip angle. $K_{\mathrm{I}}$ makes a principal contribution to the ERR distribution. The ERR increases continuously as $\alpha$ decreases, revealing that the crack grows more easily and therefore the rock has a lower tensile strength at a small dip angle. The propagation process of single cracks simulated using FRANC3D indicates that the horizontal crack grows along its initial plane. The lateral extension is quicker than the growth of wing crack; consequently, the elliptical crack grows gradually into a circular shape and finally leads to the sample fracture. For the inclined cracks, the lateral extension grows along the initial crack plane, and the wrapping wing crack propagates initially in the direction approximately perpendicular to the crack plane and then changes to the horizontal direction. After the lateral extension exceeds the half-length of short axis, the growth rate of wing crack surpasses that of the lateral extension. The combined propagation of the wrapping wing crack and lateral extension forms a spatially-distorted plane, which results in the overall fracture.

In models with two parallel cracks, the distribution of SIFs and ERRs exhibits different characteristics from that in single crack cases, due to the interaction between cracks. The crack interaction that varies with the crack spacing influences the values of SIFs and ERRs along crack fronts and the positions where the maximum $K_{\mathrm{I}}$ and $E R R$ exist. As the crack spacing increases, the ERR increases and reaches its maximum when $d / 2 a=1.25$ (the critical value), and then decreases after $d / 2 a$ exceeds 1.25. At small spacing, wing cracks in the rock bridge zone grow sufficiently and coalesce together to cause the overall fracture. After the crack spacing exceeds the critical value, those two cracks propagate separately due to the weak interaction.

The numerical results correspond well with the laboratory experimental results, except for the slight difference in the critical spacing values, which may be due to the material's heterogeneity. The distribution of mixed-mode $S I F \mathrm{~s}$ and ERRs along crack fronts and the crack growth process give an interpretation for the mechanical and failure behavior of samples. The research results can help better understand the 
growth mechanism of 3-D cracks under tension as well as the effects of crack's geometrical characteristics.

\section{References}

Ashby, M. F., and Hallam, S. D. (1986). "The failure of brittle solids containing small cracks under compressive stress states." Acta metallurgica, 34(3), 497-510.

Atkinson, B. K., and Meredith, P. G. (1987). Experimental fracture mechanics data for rocks and minerals, in Fracture Mechanics of Rock (ed. Atkinson, B. K.), Academic Press, London, England.

Ayhan, A. O. (2007). "Mixed mode stress intensity factors for deflected and inclined corner cracks in finite-thickness plates." Int. J. Fatigue, 29(2), 305-317.

Barlow, K. W., and Chandra, R. (2005). "Fatigue crack propagation simulation in an aircraft engine fan blade attachment." Int. J. Fatigue, 27: 1661-1668.

Bobet, A. (2000). "The initiation of secondary cracks in compression." Eng. Fract. Mech., 66(2), 187-219.

Bobet, A., and Einstein, H. H. (1998). "Fracture coalescence in rock-type materials under uniaxial and biaxial compression." Int. J. Rock Mech. Min. Sci., 35(7), 863-888.

Brace, W., and Bombolakis, E. (1963). "A note on brittle crack growth in compression." J. Geophys. Res., 68(12), 3709-3713.

Carter, B. J., Wawrzynek, P. A, and Ingraffea, A. R. (2000). “Automated 3D crack growth simulation.” Int. J. Numer. Meth. Eng., 47(1-3), 229-253.

Cornell Fracture Group. (2003). FRANC3D concepts \& users guide. Cornell University, Ithaca, NY, http://www.cfg.cornell.edu/.

Dong, C. Y., and De Peter, C. J. (2002). "Numerical modeling of crack reorientation and link-up." Adv. Eng. Software, 33(7-10), 577-587.

Dyskin, A. V., Jewell, R. J., Joer, H., Sahouryeh, E., and Ustinov, K. B. (1994). "Experiments on 3-d crack growth in uniaxial compression." Int. J. Fract., 65(4), R77-83.

Dyskin, A. V., Germanovich, L. N., and Ustinov, K. B. (1999). “A 3-D model of wing crack growth and interaction." Eng. Fract. Mech., 63(1), 81-110.

Dysikin, A. V., Sahouryeh, E., Jewell, R. J., Joer, H., and Ustinov, K. B. (2003). "Influence of shape and locations of initial 3-D cracks on their growth in uniaxial 
compression.” Eng. Fract. Mech., 70(15), 2115-2136.

Farmer, I. W. (1983). Engineering behaviour of rocks, 2nd Ed., Chapman \& Hall, London, England.

Hsien-Yang, Y., and Chang H. K. (1995). "Fracture mechanics of the angled elliptic crack under uniaxial tension.” Eng. Fract. Mech., 50(1), 103-110.

Lajtai, E. Z. (1974). "Brittle fracture in compression.” Int. J. Fract., 10(4), 525-536.

Li, S. C., Yang, L., Li, M. T., and Zhang, N. (2009). "Influences of 3D internal crack dip angle on tensile mechanical properties and fracture features of rock-like material." Chinese Journal of Rock Mechanics and Engineering, 28(2), 281-289. (in Chinese)

Mi, Y., and Aliabadi, M. H. (1994). "Three-dimensional crack growth simulation using BEM." Comput. Struct., 52, 871-878.

Palaniswamy, K., and Knauss, W. G. (1972). "Propagations of a crack under general in-plane tension.” Int. J. Fract. Mech., 8, 114-117.

Rahman, M. K., Hossain, M. M., and Rahman S. S. (2000). “An analytical method for mixed-mode propagation of pressurized fractures in remotely compressed rocks." Int. J. Fract., 103, 243-258.

Rizzo, F. J. (1967). “An integral equation approach to boundary value problems of classical elastostatics." Quart. Appl. Math., 25, 83-95.

Sih, G. C. (1991). Mechanics of Fracture Initiation and Propagation. Kluwer Academic Publishers, Culembourg, Netherlands.

Singh, R., Carter, B. J., Wawrzynek, P. A., and Ingraffea, A. R. (1998). "Universal crack closure integral for SIF estimation.” Eng. Fract. Mech., 60(2), 133-146.

Sousa, J. L., Martha, L. F., Wawrzynek, P. A., and Ingraffea, A. R. (1989). "Simulation of non-planar crack propagation in structures of concrete and rock." In Fracture of Concrete and Rock: Recent Developments (ed. Shaw, S. P., Swartz, S. E., and Barr, B.), Elsevier Applied Science, London, England, 254-264.

Wong, R. H. C., Law, C. M., Chau, K. T., and Zhu, W. S. (2004). "Crack propagation from 3-D surface fractures in PMMA and marble specimens under uniaxial compression.” Int. J. Rock Mech. Min. Sci., 41(3), 360-366.

Wong, R. H. C., Guo, Y. S., Liu, L. Q., Liu, P. X., and Ma, S. P. (2008). "Nucleation and growth of anti-wing crack from tips of strike-slip flaw." Proc., 42nd U.S. Rock Mechanics Symposium, San Francisco, CA, 221-321.

Wu, H. C., and Chang, K. J. (1978). “Angled elliptic notch problem in compression 
and tension." J. appl. Mech., 45, 258-262.

Wu, H. C., Yao, R. F., and Yip, M. C. (1977). "Experimental investigation of the angled elliptic notch problem in tension.” J. appl. Mech., 44, 455-461.

Yang, L., Jiang, Y., Li, B., Li, S. C., and Li, M. T. (2010a). "Influence of 3D internal crack spacing on strength and crack propagation pattern in rock sample subjected to tensile stress." Proc., 44th U.S. Rock Mechanics Symposium, Salt Lake City, Utah, $10-374$.

Yang, L., Li, S. C., Guo, Y. S., Zhu, W. S., and Lin, C. J. (2010b). "Influence of space between 3D-cracks on mechanical feature of mortar material." Rock and Soil Mechanics, 31(1), 32-38. (in Chinese)

Yang, L., Jiang, Y., Li, S. C., and Li, B. (2011). "Research on the propagation pattern of 3-D initial crack in rock-like material under uniaxial tension." Key Eng. Mater., 452-453, 817-820. 